\title{
Classification of Vibration Signal to Detect Pump Cavitation using Discrete Wavelet Transform
}

\author{
Ramadevi.R, Ph.D \\ Professor / EIE, \\ Sathyabama University, \\ Chennai - 600119, India.
}

\begin{abstract}
This paper concentrates on cavitation detection using discrete wavelet transform by classifying the pump vibration signal. Vibration signal acquired from centrifugal pump cavitation test rig carry more information about the cavitation classes. In this paper two classes has been defined namely, no cavitation class and developed cavitation class. This method uses the deviation from zero mean value of detailed components of wavelet coefficients, obtained from five level decomposition of vibration signal to detect the signal belongs to normal class or cavitation class in centrifugal pump. The main advantage of this proposed algorithm is it requires no training. In addition to this advantage a more robust results show that this algorithm has better detection response.
\end{abstract}

\section{General Terms}

Condition monitoring, Fault Diagnosis, Signal Analysis

\section{Keywords}

Cavitation, Cavitation test rig, Discrete Wavelet Transform, Decomposition Levels

\section{INTRODUCTION}

In industries, centrifugal pumps are the most widely used device than others, because of its robustness and wide ranging suitability. One of the severe problems developed in centrifugal pump is cavitation. Cavitation is a vaporization process that involves heat and mass transfer, the physical properties of the liquid and its vapor, and the flow conditions can affect the process, and thus the performance of hydraulic equipment as well[1]. This increasing critical role in industrial processes underscores the level of attention given to detection or diagnosis of potentially destructive faults like cavitation.

Many research literatures extensively documented the methods for prediction and detection of cavitation, in many of these methods vibration signal is used for the analysis purpose. Saeid Farokhzad etal.,[2] mentioned both vibration and acoustic signals carry rich and useful information about the condition of the system and it has been very popular for condition monitoring and early fault detection of pumps. Hence, vibration sensor is used here to measure the pump vibration signal.

In this research, experimental studies and analysis are carried out on centrifugal pump, to detect classes of cavitation using vibration analysis. Wavelet transform are widely used for classification and fault diagnosis. This paper presents a novel method of detection of pump cavitation system. The method used is wavelet analysis to classify cavitation vibration signal as no cavitation and developed cavitation class. Specifically mean of wavelet coefficients is used for this purpose. The main advantage of this algorithm is frequency information of pump do not have to be known in advance as in FFT algorithm.

\section{EXPERIMENTAL SETUP AND DATA COLLECTION}

The schematic illustration of experimental test loop is shown in Figure 1. The core component of the test rig facility is the pumping system. It contains a centrifugal pump with the suction side connected to the circularclosed reservoir tank and discharge side is routed back to the reservoir tank to form a closed-loop flow system. This setup has facility to induce cavitation by Net Positive Suction Head (NPSH) 3\% drop [3] method, which is fabricated at Sathyabama University, Chennai, India. The parameters that affect cavitation are identified and to measure these parameters suitable measurement devices are installed at the appropriate locations.

A state-of-the-art study has shown that vibration signal based approaches are largely used for cavitation detection in hydraulic components[4,5]. Many published methodologies use vibration and noise as features to detect the cavitation, moreover vibration monitoring is probably a convenient and a worldwide accepted method in various industries. Therefore an accelerometer is installed at various locales of the centrifugal-pump cavitation test-loop so as to collect the vibration signal.

Vibration signal is acquired from the cavitation test-loop for various suction conditions by varying vacuum pressure. The vacuum pressure is varied by means of a vacuum pump which is connected with partially filled circular tank. Both cavitation and non-cavitation signals are collected, high impulsive spikes are prominently observed in the cavitation signal.

The research data base is obtained by varying vacuum pressure using vacuum pump above the liquid in the reservoir tank, that is the suction conditions are varied. Vibration signal acquired from suction side accelerometer of the test loop for various vacuum conditions are as shown in Table 1 at 50,000 lph flow rate.

Table 1. Research Data Base

\begin{tabular}{|c|c|c|c|}
\hline $\begin{array}{l}\text { S. } \\
\text { No }\end{array}$ & $\begin{array}{c}\text { Sensor } \\
\text { Position }\end{array}$ & $\begin{array}{l}\text { No. of } \\
\text { signals }\end{array}$ & $\begin{array}{l}\text { Description of Signals } \\
\text { (at various vacuum } \\
\text { conditions) }\end{array}$ \\
\hline 1 & $\begin{array}{l}\text { At } \\
\text { suction } \\
\text { side }\end{array}$ & 14 & $\begin{array}{c}0,50,100,150,200,220 \\
240,260,280,300,320 \\
340,360 \text { and } 380 \\
\mathrm{~mm} \text { of } \mathrm{Hg}\end{array}$ \\
\hline
\end{tabular}




\section{FEATURE EXTRACTION AND DETECTION USING DISCRETE WAVELET TRANSFORM}

Multi-resolution analysis based on DWT has been used for signal analysis in a variety of applications. With the wavelet-based approach adapted, a simple quantification procedure is proposed to detect the cavitation classes in centrifugal pump by performing a multi-resolution analysis of the vibration signal, based on DWT. Vibration signal acquired from test loop for various suction conditions is decomposed into several frequency levels by discrete wavelet transform with $\mathrm{db} 4$ as mother wavelet. Discrete Wavelet Transform is applied to expose the hidden information in the cavitation signal [6]. The db4 wavelet has been chosen because of its simplicity. The extracted coefficients provide a compact representation that shows the energy distribution of the signal in scale and frequency. To reduce the dimensionality of the extracted feature vectors, mean based analysis of the wavelet coefficients are utilized [7].

A window size of 65,536 samples at $200 \mathrm{kHz}$ sampling rate is used as input to the feature extraction. The MATLAB ${ }^{\mathrm{TM}}$ program is written to decompose the vibration signal and also to extract the mean value from detail component coefficients. These values in each subband are used as a feature, which provide information about the frequency distribution of the signal. Initially the maximum decomposition level has been computed. In this case, the maximum level is five; hence five levels of decomposition are performed.

The mean as a feature is extracted from the wavelet coefficients and compared with the cavitation and nocavitation signals to detect the stages of cavitation. In this research, instead of typical checking of the mean value of vibration signal the mean value of detailed components at fifth decomposing level signal is checked for its minuscule changes. The mean values are obtained for all $\mathrm{d} 1$ to $\mathrm{d} 5$ signal and showed in Table2. From the tabular column, it is observed that the mean values of fifth level signal also changed over scale.

The mean of wavelet coefficients obtained from different decomposed signal windows refer to various classes of cavitation. This type of analysis can improve the capability of diagnosing the cavitation classes of centrifugal pumps.

\section{RESULTS AND DISCUSSION}

Cavitation classes are detected based on mean value of detail components of wavelet coefficients with respect to deviation from zero mean. These wavelet coefficients are able to pick up the small vibration disturbance caused by the cavitation. The mean values of detailed components at all five levels are extracted and fifth level values are tabulated in Table 2 also shown as graph in Figure 2. Figure 2 shows the mean value of detailed component coefficients at $5^{\text {th }}$ level decomposition using db4 wavelet for various vacuum conditions. The dependence of mean with various cavitation classes are studied from the graphs. A strong correlation was found between cavitation classes and mean value of detail components, seeing as there is a significant difference found among the vibration signal of both the cavitation classes.

From the graph it is observed that the mean value of $\mathrm{d} 1$ signal (dark blue) is almost very close to zero mean line for all vacuum conditions. Similarly in case of $\mathrm{d} 2$ (red) and d3 (green) signal, very very small deviation from zero mean line is observed for all vacuum conditions. But in the case of d4 (purple) and d5 (light blue) signal, there is small deviation from zero mean for 0 to $200 \mathrm{~mm}$ of $\mathrm{Hg}$ vacuum conditions and much deviation from 220 to $380 \mathrm{~mm}$ of $\mathrm{Hg}$ vacuum conditions. Based on these observations author can conclude that because of the high impulsive spikes present in the cavitation signal the mean values detail components are also changed.

This indicates 0 to $200 \mathrm{~mm}$ of $\mathrm{Hg}$ belongs to no cavitation class and 220 to $380 \mathrm{~mm}$ of $\mathrm{Hg}$ corresponds to cavitation class. The results of cavitation detection algorithm for pump using discrete wavelet transform technique are provided in Table 3. In this analysis, mean value of detail coefficient was considered for classification purpose. The decision on two different stages of cavitation was made with respect to deviation from zero mean. To conclude, the pump vibration signal is classified into no cavitation and developed cavitation class based on deviation from zero mean value of detail coefficients.

\section{CONCLUSION}

In this paper, the proposed methodology is mean based discrete wavelet transform algorithm for classification of cavitation signal. This algorithm examines the deviation from zero mean value of detailed dwt co-efficient at various level of both cavitation and no cavitation signals, which are collected from centrifugal pump cavitation test loop. This proposed mean based DWT algorithm was tested on over 3 different flow sets with 3 different sensor positions data samples, and detected cavitation stages with $100 \%$ reliability. Therefore it offers a reliable solution for detecting the cavitation. The results indicate that the proposed algorithm is an efficient way of classifying the various cavitation signals. The proposed DWT algorithm with $\mathrm{db} 4$ wavelet has five decomposition levels and based on deviation from zero mean value of detailed wavelet coefficient is used for detecting various cavitation stages of centrifugal pump in cavitation test loop.

This research demonstrates a comprehensive perspective of cavitation detection methods based on wavelet transform and their application in centrifugal pump cavitation test rig. The cavitation detection algorithm discussed in this paper could identify cavitation class present in pump. Detecting cavitation in a timely manner is vital to maintaining a properly functional system. Cavitation class detection task is performed with mean as a feature. The main advantages of this algorithm are it requires no training as in the case of neural network and provides a more robust solution. The wavelet analysis proves to be successful for detection of cavitation in a centrifugal pump.

Relevant classification techniques can also be used for other hydraulic equipments and also for any signal analysis. The conclusions reached as an outcome of the research work performed for this paper point to prospect avenues of analysis. Future research needs to be done to investigate under which conditions the pump generate cavitation. In order to successfully investigate this problem, a parameters based approach would be required. And also future work can focus on integrating the feature extraction efficiency of the wavelet transform with the classification capabilities of neural network for signal classification in the context of detecting the cavitation. 


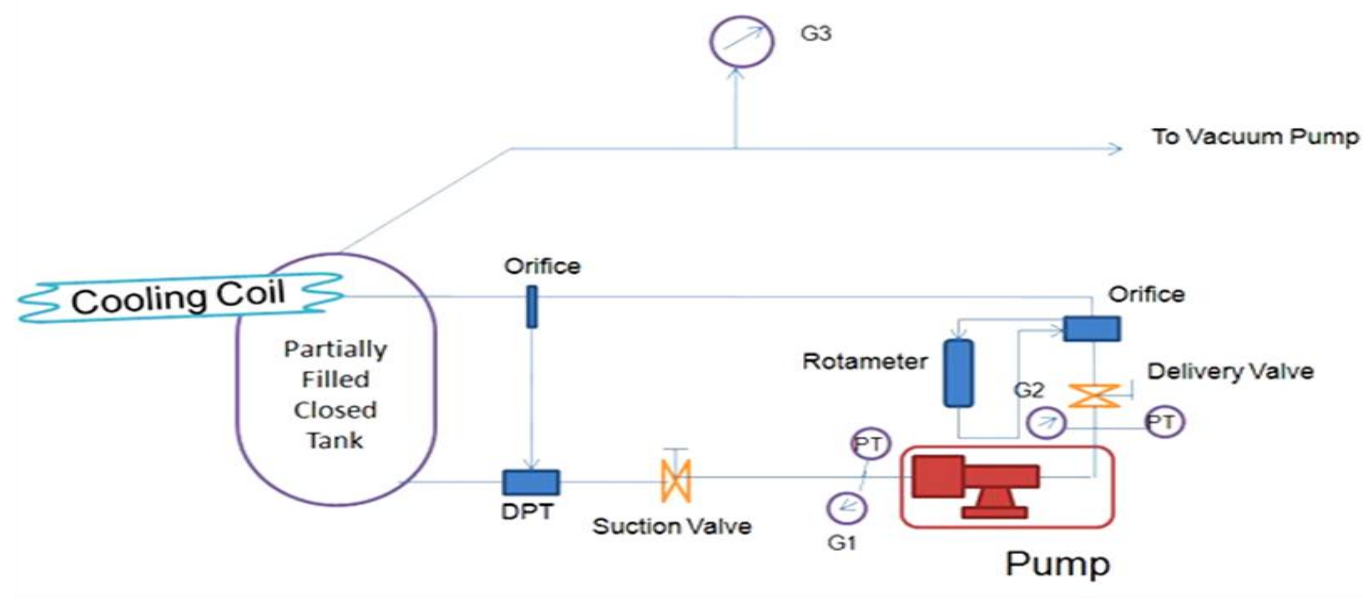

Fig 1: Schematic illustration of Experimental Test Loop

Table 2 Mean Value of db4 Wavelet Coefficients

\begin{tabular}{|c|c|c|c|c|c|}
\hline $\begin{array}{c}\text { Vacuum Pressure } \\
(\mathbf{m m} \text { of Hg) }\end{array}$ & $\mathbf{d 1}$ & $\mathbf{d 2}$ & $\mathbf{d 3}$ & $\mathbf{d 4}$ & $\mathbf{d 5}$ \\
\hline $\mathbf{0}$ & 0.001273 & 0.000312 & -0.0003197 & -0.003067 & -0.0009571 \\
\hline $\mathbf{5 0}$ & -0.001721 & -0.0002037 & 0.007633 & -0.00132 & -0.02337 \\
\hline $\mathbf{1 0 0}$ & -0.0007855 & -0.0002007 & 0.000305 & -0.003299 & -0.009273 \\
\hline $\mathbf{1 5 0}$ & -0.0002095 & 0.0009986 & 0.0001861 & 0.003006 & -0.000234 \\
\hline $\mathbf{2 0 0}$ & -0.001129 & 0.0007532 & 0.0009549 & 0.00553 & -0.02471 \\
\hline $\mathbf{2 2 0}$ & -0.0002196 & -0.0006355 & -0.0002649 & -0.01128 & 0.001588 \\
\hline $\mathbf{2 4 0}$ & -0.001154 & 0.0004873 & -0.0006655 & -0.114 & 0.08928 \\
\hline $\mathbf{2 6 0}$ & -0.0001475 & -0.000217 & 0.001353 & -0.01158 & 0.02213 \\
\hline $\mathbf{2 8 0}$ & 0.001155 & 0.0002889 & 0.002207 & -0.03594 & 0.06113 \\
\hline $\mathbf{3 0 0}$ & -0.0003482 & -0.002307 & -0.0006033 & -0.03712 & -0.1564 \\
\hline $\mathbf{3 2 0}$ & -0.0004147 & -0.001252 & -0.004605 & 0.01521 & -0.1924 \\
\hline $\mathbf{3 4 0}$ & -0.0001261 & -0.0005213 & -0.001106 & -0.006392 & -0.03711 \\
\hline $\mathbf{3 6 0}$ & -0.001296 & 0.0002093 & 0.001572 & -0.01657 & -0.06455 \\
\hline $\mathbf{3 8 0}$ & -0.001211 & 0.000832 & 0.00232 & 0.001325 & -0.0348 \\
\hline
\end{tabular}

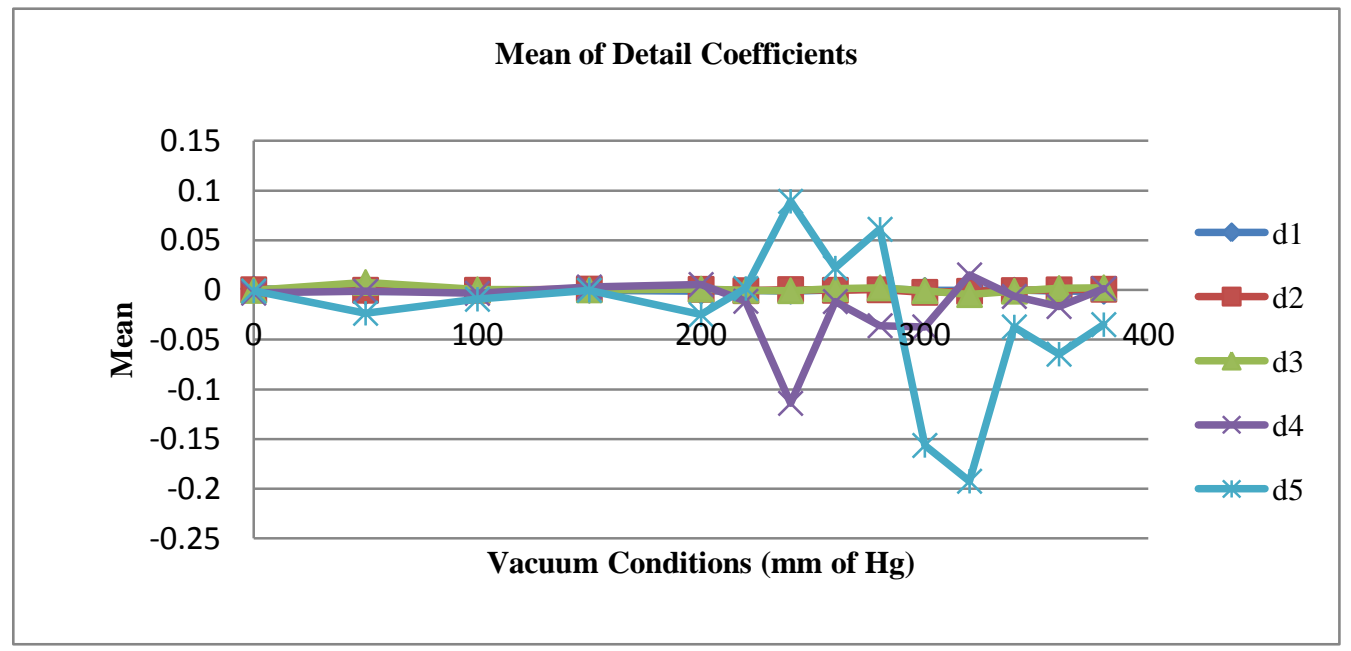

Fig 2: Mean Values of all five levels Detail Component Coefficients 
Table 3 Classification results of DWT based algorithm for db4 wavelet

\begin{tabular}{|c|c|c|}
\hline \multirow{2}{*}{$\begin{array}{c}\text { Parameters } \\
\text { Considered }\end{array}$} & \multicolumn{2}{|c|}{ Suction Conditions Classified into } \\
\cline { 2 - 3 } & No Cavitation Signals & Developed Cavitation Signals \\
\hline $\begin{array}{c}\text { Mean value of } \\
\text { Detail Components }\end{array}$ & $\begin{array}{c}0,50,100,150 \text { and } 200 \\
\mathrm{~mm} \text { of } \mathrm{Hg}\end{array}$ & $\begin{array}{c}220,240,260,280,300,320,340, \\
360 \text { and } 380 \mathrm{~mm} \text { of } \mathrm{Hg}\end{array}$ \\
\hline
\end{tabular}

\section{ACKNOWLEDGMENTS}

The author wish to thank Shri.Sreedhar B.K and Shri.Shivprakash Ruhela, the scientists of Indira Gandhi Center for Atomic Research (IGCAR), Government of India, for their technical support. And also she wants to thank Dr.SheelaRani.B, Vice chancellor, Sathyabama University for her constant support towards this research work.

\section{REFERENCES}

[1] Roggers R.S. and Moore R. D. (1969), "Method for Prediction of Pump Cavitation Performance for Various Liquids, Liquid Temperatures, and Rotative Speeds", NASA TN D-5292, NASA Scientific and Technical Publications, Lewis Research Centre, Cleveland, Ohio.

[2] Saeid Farokhzad, Naeim Bakhtyari, Hojjat Ahmadi,"Vibration Signals Analysis and Condition Monitoring of Centrifugal Pump", Technical Journal of Engineering and Applied Sciences, pp 1081 1085, Vol.3, No.12, 2013.

[3] Bruno Schiavello and Frank C.Visser (2009), "Pump Cavitation - Various NPSHR Criteria, NPSHA Margins, and Impeller Life Efficiency", Proceedings of the Twenty-Fifth International Pump Users Symposium, $23-26^{\text {th }}$ Feb 2009, Houston, Texas, USA, pp.113-144.

[4] Huaqing Wang, Peng Chen “ Sequential Condition Diagnosis for centrifugal pump system using Fuzzy Neural Network", Neural Information Processing Letters and Reviews, Vol11, No.3, March 2007.

[5] H.Q.Wang, P.Chen " Fault Diagnosis of Centrifugal Pump using Symptom Parameters in frequency Domain" Agricultural Engineering International:the CIGR Ejournal, Vol.IX, Nov 2007.

[6] Guillermo Palacios J., Ramon Beltran and Raquel Lacuesta (2005), "Multi resolution Approaches for Edge Detection and Classification based on Discrete Wavelet Transform", Discrete Wavelet Transforms: Algorithms and Applications", pp. 81-100.
[7] Yasaman Zandi Mehran, "New Application of Wavelet Transform in Classification the Arterial Pulse Signals, Proceedings of the $5^{\text {th }}$ WSEAS international Conference on system science and simulation in Engineering, Spain, Dec 2006, pp448 453.

[8] Henrique Mohallem Paiva, Roberto Kawakami Harrop Galvao, Luis Rodrigues “ A Wavelet based Multivariable Approach for fault detection in dynamic systems", Sba Controle \& Automacao, Vol.20, No. 4 Natal Oct / Dec 2009.

[9] ZHOU Hongbin, LI Hui “ Diagnosis of preliminary cavitation in pumps by wavelet analysis" Energy Resource and Power Engineering, 2010, pp 394 398.

[10] GaoY. and Ron J Patton (2003), “Applications of Wavelet Analysis for Performance monitoring and Diagnosis of a Hydraulic Pump", $5^{\text {th }}$ IFAC symposium on Fault Detection, Supervision and Safety of Technical Processes, Washington, USA, pp. $333-338$

[11] Juan Jose Gonzalez de la Rosa, Lloret I., Moreno A., Puntonet C.G. and Gorriz J.M. (2006), "Wavelets and Wavelet Packets Applied to Detect and Characterize Transient Alarm Signals from Termites", Elsevier, Measurement, Vol. 39, pp. 553-564.

[12] Mukesh Sahdev (2010), "Centrifugal Pumps: Basic Concepts of Operation, Maintenance and Trouble Shooting, Part II", The Chemical Engineering's Resource Page, WWW. Chersources. com .

[13] Paul S Addison (2005), "Wavelet Transforms and the ECG: a review", Institute of Physics Publishing Physiological Measurement 26, pp. R155-R199.

[14] Emily K.Lada, Jye - Chyi (JC) Lu, James R. Wilson "A Wavelet based procedure for process Fault Detection," IEEE Transactions on Semi Conductor Manufacturing, pp 1-31. 